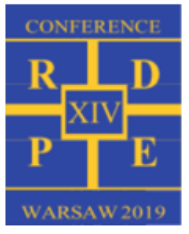

\title{
Identification of the liquid mass fraction content in the wet steam
}

\author{
Mirosław M ajkut ${ }^{1,}{ }^{,}$, Stawomir Dykas ${ }^{1}$, Krystian Smołka ${ }^{1}$ and Michał Strozik ${ }^{1}$ \\ ${ }^{1}$ Silesian University of Technology (SUT), Institute of Power Engineering and Turbomachinery, K onarskiego 18, 44-100 Gliwice, \\ miroslaw.majkut@polsl.pl,Poland
}

\begin{abstract}
The experimental research, initiated at the Institute of Power Engineering and Turbomachinery of the Silesian University of Technology at the end of the last century, created an opportunity to supplement knowledge about the phenomenon of steam flow with condensation, tested by numerical fluid mechanics by IM iUE employees. In parallel with the numerical work, significant progress has been made in experimental studies on the flow of wet steam. Recently, measuring techniques based on recording the phenomenon of extinction of light have been elaborated or improved. The basic value determined in the final stage in the experimental way was the content of the liquid phase defined as the wetness fraction. The methodology of tests and experimental investigations was presented, as well as the applied and developed measurement systems. Next, some developed designs of $X$ probe and their modifications are described. Article present al so some examples of applications of the developed measurement techniques in application to experimental research conducted on wet steam in the IMiUE. Examples of comparison between experimental and numerical tests are also provided.
\end{abstract}

\section{Introduction}

In recent years, thermal power plants have requirements to minimize their impact on the natural environment, regardless of the load and parameters of the system. Thanks to the operation experience gained over decades and progress in the field of numerical modelling of fluid flow, stress and strain states modelling in solid bodies, the efficiency of electricity generation in such power units has more than doubled. The current requirements regarding the reduction of pollutant emissions and the flexibility of steam power units, force them to continuously improve the operation of the machines and power equipment included in their structure. In the case of steam condensing turbines, which are a key element of, for example, coal-fired, steam-gas or nuclear blocks, an important element from the point of view of maintenance is the measurement of wetness mass fraction $y[\%]$ (or dryness $x=100 \%-y$ ) at the turbine outlet. There are many experimental methods used to determine the volume of the liquid phase and, as a result, also wet steam enthalpy in the turbine. These methods have been created and have been partly used since the early 1960s. Simple calorimetric methods [19] or early optical methods can be mentioned here. The introduction of various types of analyzes of the obtained flow image has now enabled not only the determination of the total value of wet steam wetness, but also enabled an accurate analysis of its composition. There are currently many measuring methods available for twophase mixtures. They are: impedance [1]), optical, using electromagnetic or ultrasonic radiation. In the case of tests conducted for opaque factors, there are methods based on Doppler shift of reflected light (LDV), or analysis of shifted images (PIV). Numerous measurement techniques using a laser beam have also been developed. These include Rayleigh scattering [31], Raman spectroscopy [31], laser induced fluorescence (LIF) [27], spectral spectroscopy [21], as well as techniques using Fraunhofer diffraction of laser radiation by droplets [26], phase Doppler anemometry (PDA) [2] or the use of laser light extinction [6]. Each technique has its advantages and disadvantages and is useful in a specific field of application. They have different temporal and spatial resolution, and some of them may require wide optical access, so practical implementation of such measurement in industrial conditions becomes difficult. A mong the methods tested so far, techniques based on the phenomenon of light extinction are of particular interest, attractive due to their relative simplicity of implementation and data interpretation, the possibility of continuous measurement with a quick time response, as well as very limited optical requirements when it comes to access to the measurement space. Optical methods use the phenomenon of wave weakening (in this case light over a wide range of wavelengths) by the associated scattering. One of the main techniques currently being developed in application for measurements in steam turbine channels by several significant research centers from Germany [17, 24], China $[4,5]$ or the Czech Republic [20] is the method based on light absorption, which uses in its basic form the theory of scattering M ie on spherical particles. 


\section{Condensation phenomenon and elements of light scattering theory}

In two-phase flow systems, the typical droplet formation process mainly begins through homogeneous or heterogeneous condensation. Phenomena lead to the creation of so-called nucleation nuclei, which are a set of molecules that grow under the conditions of the surrounding gas phase. The resulting droplets (primary and secondary) have sizes from $1 \mu \mathrm{m}$ to $100 \mu \mathrm{m}$ and high concentrations. The measured moisture content of water vapor as a two-phase mixture $y$ is usually determined from the volume fraction of droplets (liquid phase) $\mathrm{C}_{\mathrm{vl}}$ and the density of the gas phase $\rho_{\mathrm{v}}$ and liquid phase $\rho_{1}$ in the form:

$$
\begin{gathered}
y=\frac{m_{l}}{m_{m}}=\frac{m_{l}}{m_{l}+m_{v}}=\frac{C_{v_{l}} \rho_{l}}{C_{v_{l}} \rho_{l}+\left(1-C_{v_{l}}\right) \rho_{v}} \\
\mathrm{C}_{\mathrm{v}_{\mathrm{l}}}=\frac{\pi}{6} \mathrm{D}_{32}{ }^{3} \mathrm{~N}
\end{gathered}
$$

where:

$D_{32}$-Sauter diameter

$N$ - number of droplets

In the last several years, numerical modelling of steam flow with condensation in the final stages of turbines has achieved significant progress $[7,8,22,23,29]$ and now these methods enable turbine designers and scientists to fully simulate the processes of supercooling, nucleation and droplet growth in the considered area research. However, the current advancement of these methods and the relatively small effort, needed to obtain the results of numerical simulations of condensation of steam flow, often overshadows the fact that the calculations are essentially based on mathematical models, e.g. nucleation and drop growth. As a consequence, at least some of the results of computational fluid dynamics must be validated against experimental studies to verify their accuracy.

Such tests are often carried out in laboratory channels with measuring elements in the form of various types of nozzles, in which the steam is continuously expanded from the conditions in the expansion tank installed at the inlet to a constant pressure in the outlet chamber, and the condensation process occurs during expansion in the channel. A part from the instability of the flow, which may result from the occurring heat losses, there are usually no additional disturbances caused, for example, by the presence of blade channels in the actual machine. Also taking into account the fact that, even for nozzle flows, the results of numerical analyzes often differ from the results of experimental tests, especially with respect to droplet sizes and their concentration, it is necessary to further confirm the numerical results for flows with steam condensation in turbines by means of experimental tests. In this regard, it should be mentioned that although the previously determined vapor wetness level is an important parameter for assessing the quality of flow calculations, the droplet size distribution and diameter is the main criterion, since the wetness level itself is usually determined by the thermodynamic conditions at the beginning of the condensation process.

Depending on the geometrical relationships and the wavelength of light, two types of scattering are mainly distinguished: Rayleigh and Mie [18]. There are also other specific types, such as scattering on very large particles, called geometric (Fig. 1).

The main scattering parameters are:

- wavelength of the incident radiation $\lambda$,

- scattering particle size, usually expressed as dimensionless parameter $X=\frac{2 \pi r}{\lambda}$,

where: $r$ - radius of the spherical scattering particle,

- quantity taking into account optical properties, i.e. the refractive index.

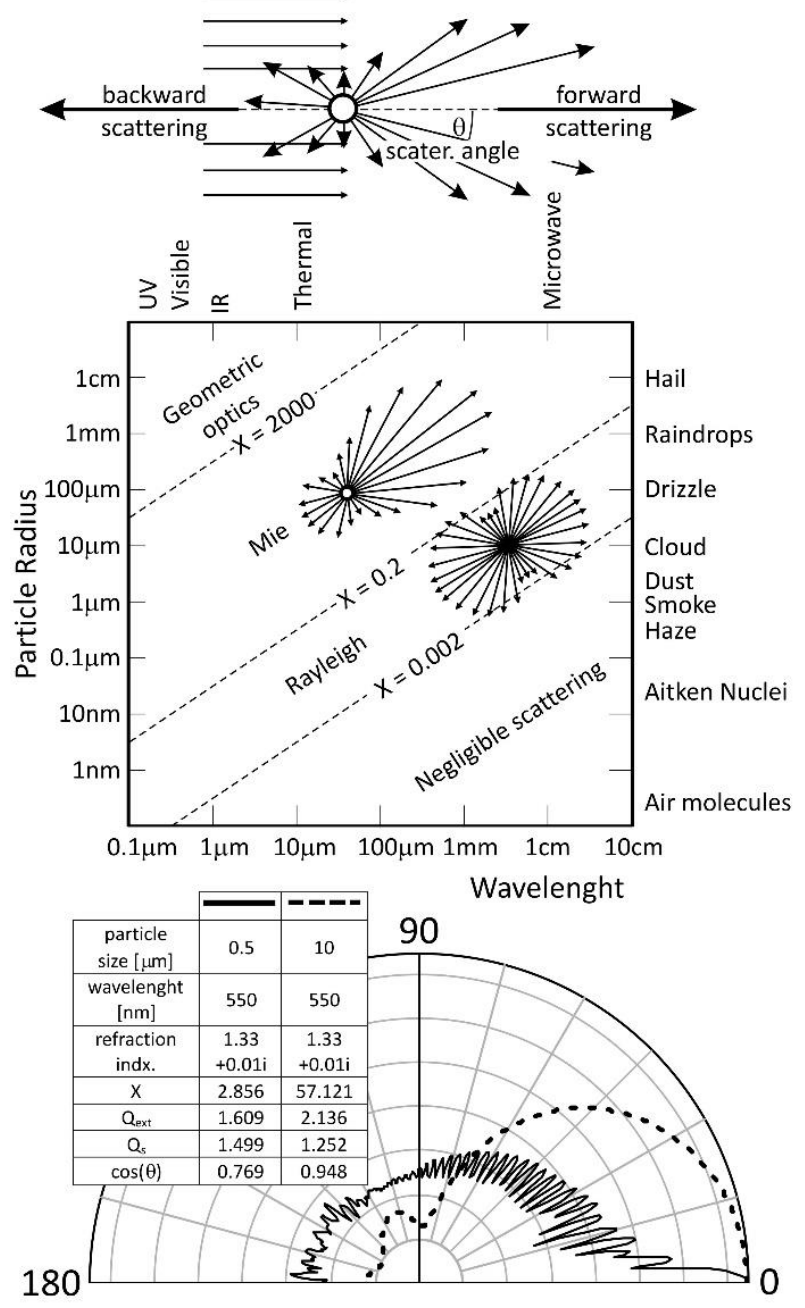

Fig. 1. Light scattering regimes and scattering geometry as well as exemplary scattering curves for two different particle diameters (obtained according to the IM iUE procedure)

As can be seen from the (Fig. 1), in the case of increasing the diameter of the scattering particles, we are dealing with a change in the scattering symmetry to an increased forward scattering. We are dealing here with the $\mathrm{M}$ ie effect. Its main essence is the assumption of particle sphericity and its homogeneity, therefore a single refractive index at a given wavelength can be assumed. However, it requires the adoption of a relative refractive index, which is the refractive index of the particle divided by the refractive index of the medium. 
For water, the value is usually taken as $n=1.333$, but this value also depends on the wavelength of the incident light and temperature.

\section{Dependencies regarding extinction method}

In the method of light absorption (extinction), the wavelength $\lambda$ and the original intensity $\operatorname{I} 0(\lambda)$, after passing the distance $L$ through the medium of homogeneous particles (drops), is reduced to the intensity $\mathrm{I}(\lambda)$ (Fig. 2).

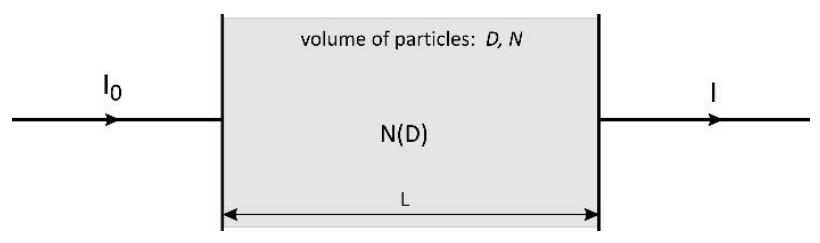

Fig. 2. Scheme of attenuation of the primary intensity lo after crossing the distance $L$ in the light extinction method
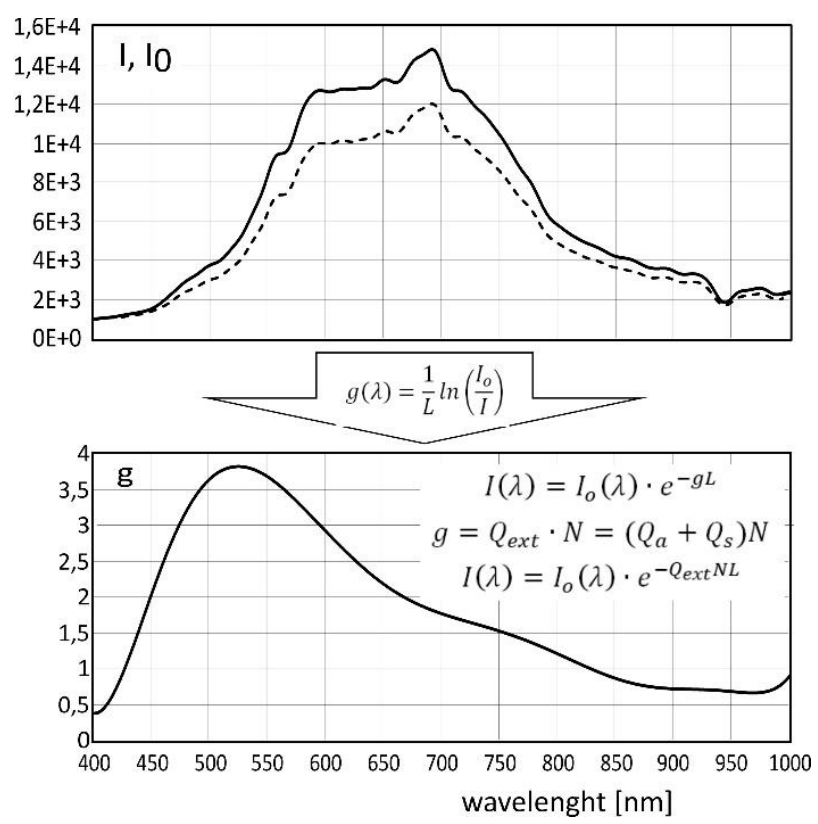

Fig. 3. Light intensity curves I and Io (experimental measurement) and the curve of the extinction coefficient $g$ as a function of the wavelength of light

In the case of the considered method, considering the dependence of both intensities on the wavelength and marking the optical thickness as $\xi$, it can be written that:

$$
I(\lambda)=I_{0}(\lambda) \exp [-\xi(\lambda)]
$$

For the homogeneous distribution of particles, the modified Bouger-L ambert-B eer equation (which is used in absorption spectroscopy) takes the form [32]:

$$
\frac{1}{t} \ln \left(\frac{I(\lambda)}{I_{0}(\lambda)}\right)=\frac{\pi}{4} \int_{D_{\min }}^{D_{\max }} Q_{\text {ext }}(n, D, \lambda) N(D) D^{2} d D
$$

In most cases, we use the Sauter diameter in comparisons, which we define as follows for the above considerations:

$$
D_{32}=\frac{\int_{D \min }^{D \max } D^{3} N(D) d D}{\int_{D \min }^{D \max } D^{2} N(D) d D}
$$

The determination procedure for the case adopted in IMIUE consists in the first phase of determining the dependencies resulting from the B ouguer-L ambert-B eer law for the entire measured wavelength range of light. Fig. 3 presents this principle graphically, obtaining a curve of the dependence of the extinction coefficient from the measurement depending on the wavelength.

Then, using the dependencies according to M ie@ law, the $Q_{\text {ext }}$ coefficient and the final form of the equation were determined according to

$$
g(\lambda)=\frac{1}{L} \ln \left(\frac{I_{o}}{I}\right)
$$

and

$$
g(\lambda)=\int_{D_{\min }}^{D_{\max }} \frac{\pi}{4} D^{2} \cdot Q_{\text {ext }}(D, \lambda, n) \cdot N(D) d D
$$

marking as $K_{j, i}$ the part of the above dependence taking into account the droplet dimensions:

$$
K_{j, i}=\frac{\pi}{4} D_{j}^{2} \cdot Q_{e x t}\left(D_{j}, \lambda_{i}, n_{i}\right) \Delta D
$$

and as $f_{j}$ quantities regarding their numbers:

$$
f_{j}=N\left(D_{j}\right)
$$

we can save the form of the pattern as:

$$
\left(\begin{array}{c}
g\left(\lambda_{1}\right) \\
\vdots \\
g\left(\lambda_{m}\right)
\end{array}\right)=\left[\begin{array}{ccc}
K_{1,1} & \cdots & K_{1, n} \\
\vdots & \ddots & \vdots \\
K_{m, 1} & \cdots & K_{m, n}
\end{array}\right] \cdot\left(\begin{array}{c}
f\left(D_{1}\right) \\
\vdots \\
f\left(D_{n}\right)
\end{array}\right)
$$

in other words: $g_{i}=K_{j, i} \cdot f_{j}$

in equation (10) $g_{i}$ is a vector determined in the course of direct measurement on a spectrometer, $K_{j, i}$ is a matrix calculated using $\mathrm{M}$ ie procedures, and function $f_{j}$ is a vector that requires determination. In this case, we have to solve the inverse problem, in which it is necessary to determine the inverse matrix $K_{j, i}{ }^{-1}$, i.e. solve the equation:

$$
f_{j}=K_{j, i}{ }^{-1} \cdot g_{i}
$$

Considering the curve in the drawing (Fig. 4), showing an example of the course of $Q_{\text {ext }}$ variability depending on the Mie - $X$ parameter, plotted in logarithmic coordinates, it can be seen that for the range $1<X<2.5$ the curve runs almost linearly, so that it is possible to determination of the inverse matrix. However, for larger particles, the larger the number of populations and the more dispersed their sizes are, the more it is impossible to determine by standard methods the inverse matrix [25]. The main problem in this case is the stability of the vector $g_{j}$ for the measured wavelength range of light. 
For this reason, it is proposed to use the smoothing curve $g_{j}$ or to determine the size of $I_{o}$ and $I$ in a way that allows obtaining the vector $g_{j}$ with the smallest possible standard deviation for the entire spectrum.

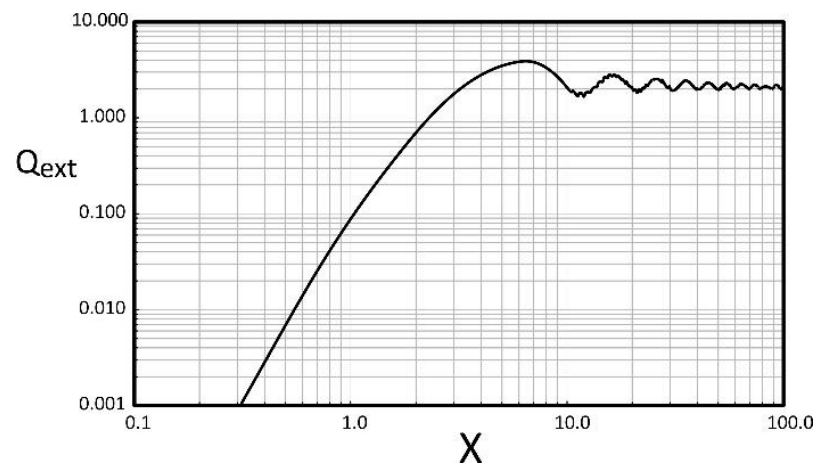

Fig. 4. Extinction coefficient as a function of the $M$ ie parameter $X$ for $m=1.33+0.0 \mathrm{i}$

In the literature [25], several methods can be found to solve this problem. The most common is the method presented by Philips and Twomey [28] as well as Lawson and Hanson [68], using the classic NNLS (nonnegative least squares) approach. The N N LS method is a regression method that guarantees obtaining only positive (or zero) coefficients and solves equation (11) as:

$$
\min _{f}\|K \cdot f-g\|^{2}, \quad \text { where } f \geq 0
$$

\section{Construction of $X$ probe}

Recently, optical methods, based on the technique of light absorption, together with the development of technology, give increasingly accurate and reproducible results. The most important of the advantages of this method can be formulated as follows:

- it is possible to obtain not only information about the wetness level of the steam, but also about the size and concentration of liquid phase droplets,

- fiber optic probes are treated rather as non-invasive (or minimally invasive) due to the transparency of the measuring space in which only light is located,

- scattered light data analysis is usually conducted for the visible wave range $(0.45-0.80 \mu \mathrm{m})$, so you can use simple and relatively cheap optics and spectroscopic systems,

- measurements can be made directly inside the device. No sampling is necessary. Local thermodynamic parameters are not disturbed,

- the measurement is fast, possible to do in a few seconds or less

- optical probes can be built into long holders with a circular cross section with a small outer diameter. Therefore, they can be used to make measurements not only outside the last turbine stage, but also between subsequent stages. Such a handle also allows for quite simple integration with machine structure.

The $X$ probe measuring system at IMIUE includes elements of the lighting system in the form of a deuterium-halogen illuminator and a spectrometer, as well as a traverse system. In addition, probe software was created to automate the measurement process and conduct on-line signal analysis.

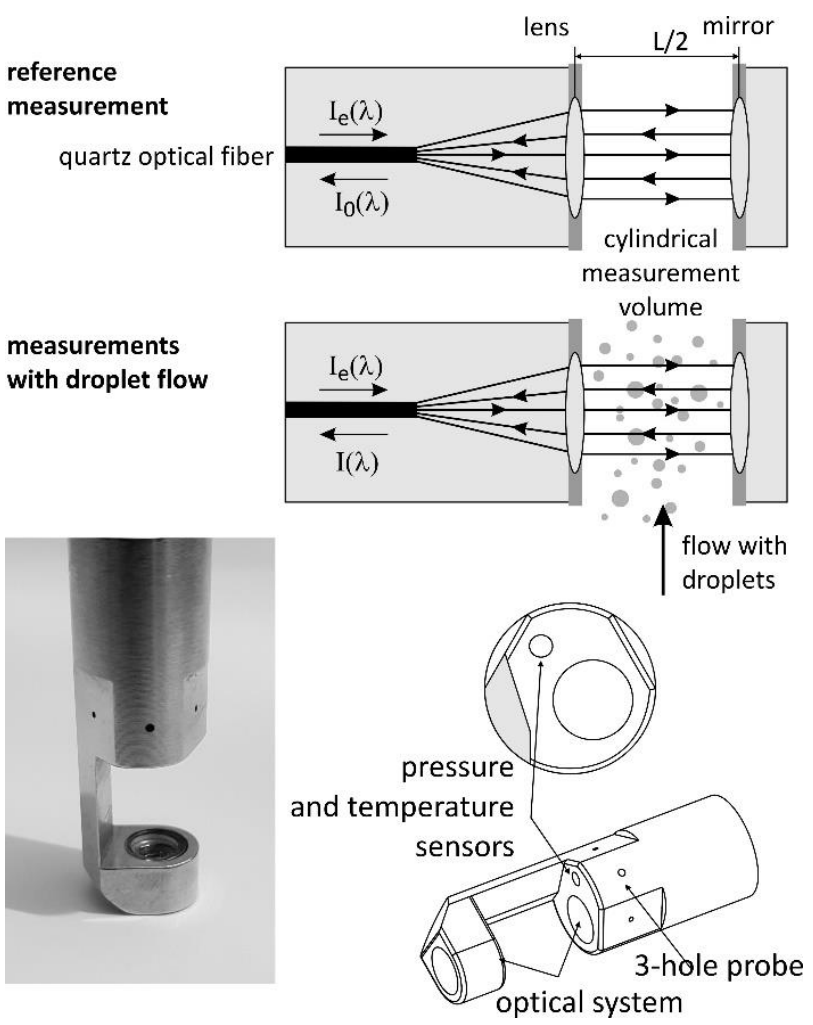

Fig. 5. Determination of light extinction coefficient by measurement of reference intensity and attenuated intensity and view of the current version of the $X$ probe (IM iUE)

For the correct determination of the size and number of drops, as well as for a full description of the stream parameters, it is necessary to know the basic thermodynamic parameters of the tested flow. The probe design has implemented a 3-hole probe for measuring several temperatures and pressures, as well as to determine the angle of the flow in relation to the measuring space. The part of the probe responsible for determining the flow angle was placed in the upper part of the sensor, on the element having the triangle crosssection. The probe was aerodynamically optimized, reducing the sensor diameter to $10 \mathrm{~mm}$, making it less invasive (Figure 5).

\section{Description of the $X$ probe measuring system}

The $X$ probe measuring system (Fig. 6) consists of two main blocks. The first of them is responsible for the production, registration and analysis of the light spectrum, while the second for measurements of thermodynamic parameters. The illuminator used for testing was equipped with two types of light sources enabling the production of a collimated beam both in the visible range ( $400 \mathrm{~nm}$ to $2000 \mathrm{~nm}$ ) and extended by the ultraviolet range ( $200 \mathrm{~nm}$ to $400 \mathrm{~nm}$ ) (Fig. 6).

Pressure registration was carried out using highprecision $0.16 \%$ transducers from Applisens. The figure 6 presents a view of the $X$ probe measurement 
system during tests in the steam tunnel and the spectrum of the lamps.
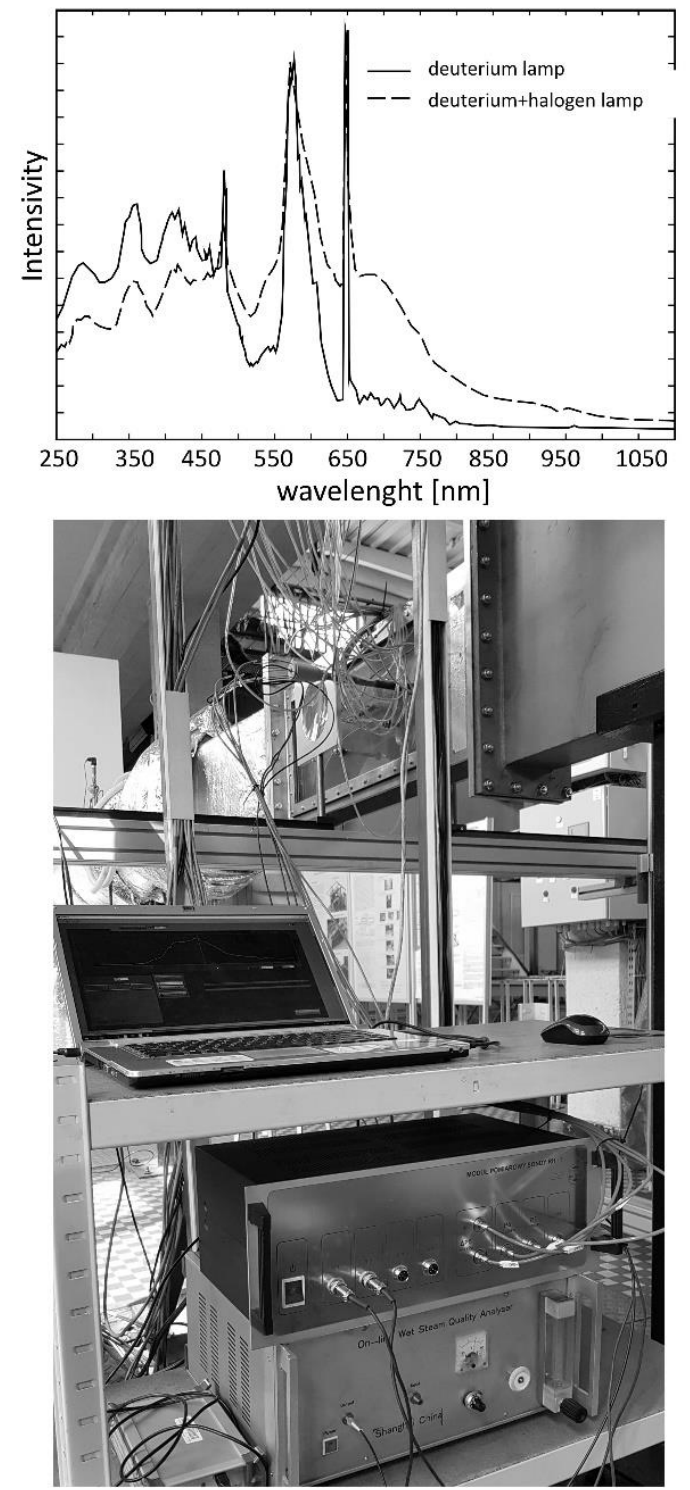

Fig. 6. $V$ iew of the $X$ probe measurement system during tests in the steam tunnel and the spectrum of the deuterium and deuterium + halogen lamp

The test system, prepared and checked for correct operation, was subjected to test and calibration measurements in the medium stream with known droplets diameter and its concentration, as well as in a specially prepared vacuum test chamber. The pressure recording system has been calibrated before being placed in the tunnel in accordance with the procedure enabling determination of appropriate calibration factors.

\section{Static and comparative tests of the proposed light extinction system}

In the first phase, the values of droplet diameter and their concentration were determined using static tests. For this purpose, polystyrene particles with a diameter $D \cong$ $2 \mu m \pm 0.05 \mu m, N=1.210^{11} 1 / \mathrm{m}^{3}$, suspended in an aqueous medium, were used. The figure (Fig. 7) presents the view of the probe during the tests, as well as the light spectrum $I$ and $I_{o}$ along with the graph of particle diameter distribution and their numbers.
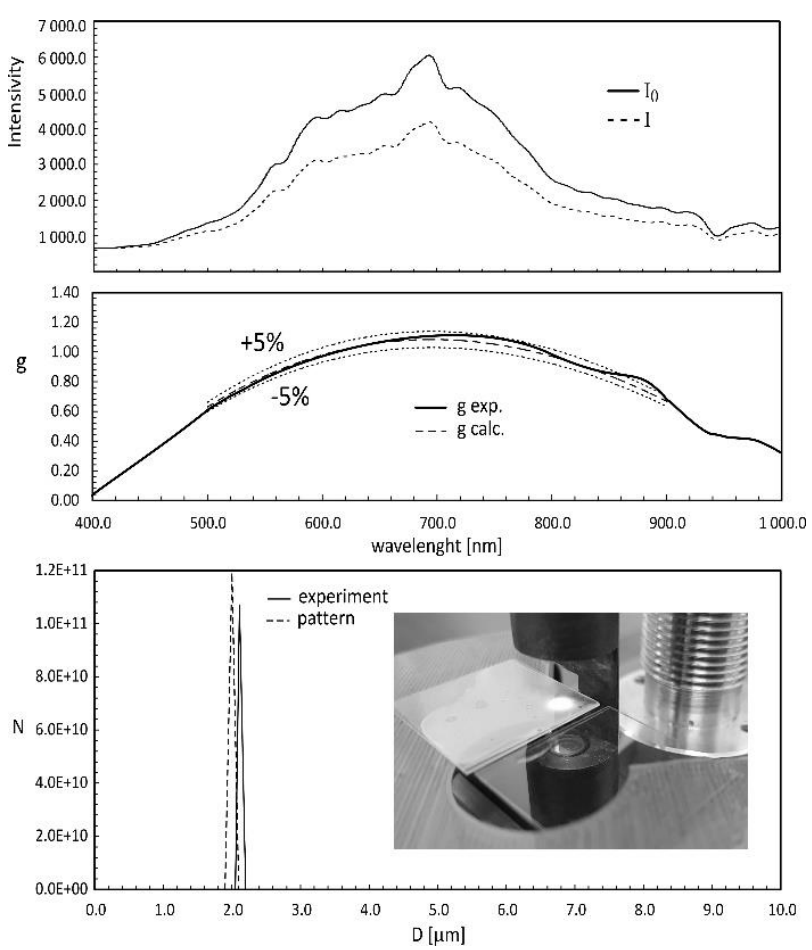

Fig. 7. $I$ and $I_{o}$ light spectrum, distribution of extinction coeff. $g$ from measurement and calculations, distribution of particle diameter and their number, view of $X$ probe during tests

The figure 7 compares the distribution of the extinction coefficient $g(\lambda)$ determined during test measurements with the coefficient obtained from the relationship (7). In addition, curves showing the relative error $( \pm 5 \%)$ of the calculated value were marked on the graph. Satisfactory test results for polystyrene particles were achieved, for which the error was $5.75 \% \quad(2.115 \mu \mathrm{m}$ - value determined during the test) and the number of particles was $12.1 \%\left(1.06 \times 10^{11} 1 / \mathrm{m}^{3}\right)$. The larger error in the number of determined particles could have been caused by the difficulty associated with the correct estimation of their number in the measurement volume. In addition, the test results obtained and the calculation procedure itself were subjected to additional verification, checking them against the procedure developed at the Technical University in Stuttgart. Researchers at this University have also developed their own measurement procedure, based on the method of light extinction, proven many times in a large number of different type of tests, as well as in tests carried out in a real turbine or using numerical analysis [17, 24].

The comparative test measurement was based on data obtained from the actual steam flow during tests in the nozzle installed in the IM IUE steam tunnel. Extensive research on this stand, conducted with the use of wet steam and humid air, has been described in co-author monographs [16] and in numerous publications [9-13]. For critical analysis of results, a case was selected for which the results of the comparison of procedures were presented in the figure 8. 


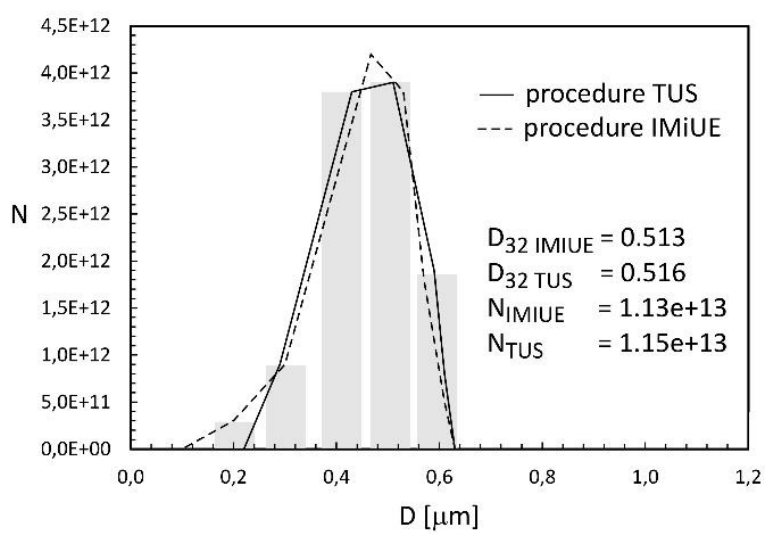

Fig. 8. Distributions of droplet diameter and their numbers presented for the IM IUE and TUS procedure

Comparative studies have shown high compliance of the proposed calculation procedures. The size deviations determined for the interesting wavelength range of light after optimization of the IM iUE system did not exceed $0.3 \%$ for the droplet diameter, and $1.7 \%$ for their number, using identical input data.

\section{Determination of the wetness and the diameter of the droplets for the real wet steam flow at the outlet from the steam tunnel nozzle}

From the point of view of the practical application of the presented test method, tests carried out under real conditions of wet steam flow in a steam tunnel give the largest view on its measuring capabilities. The possibility of comparing experimental measurements with advanced numerical fluid mechanics methods carried out in parallel is also valuable. For this purpose, the $X$ probe was used to tests carried out in a research steam tunnel located in the IMiUE Thermal Machinery $\mathrm{Hall}$, used for a wide spectrum of steam flow tests, using various types of nozzles and blade channels. Research using the installation has been extensively described in publications over the last several years [14-16]. To carry out the tests, a measuring nozzle with the geometry shown in the figure 9, [14], and the A nsys CFX software were used. Solver $\odot$ criteria and other relevant code settings have been set in accordance with Ansys recommendation for wet steam flow calculations, and detailed description of the model used, can be found in the literature [30]. The obtained results of numerical analyzes were compared with the results obtained at the laboratory, i.e. with the distribution of static pressure along the nozzle, the image of the flow field obtained using the Schlieren technique and, most importantly, with the measured wetness and the Sauter diameter, determined at the outlet of the measuring nozzle. A more detailed description of the experimental research object and the complementary measuring techniques used in the experiment were presented in publications [3, 10, 12].

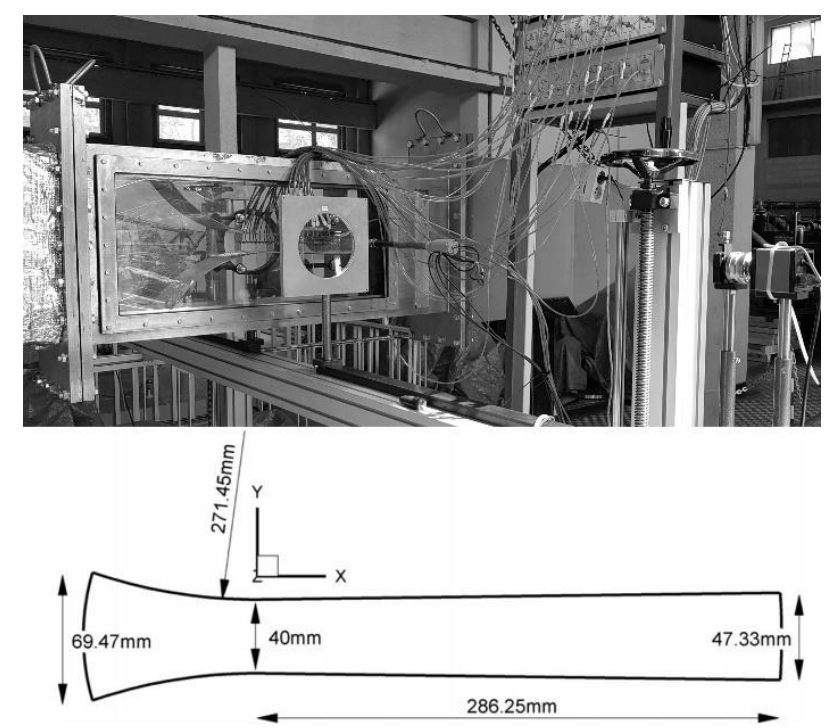

Fig. 9. The geometry of the measured nozzle
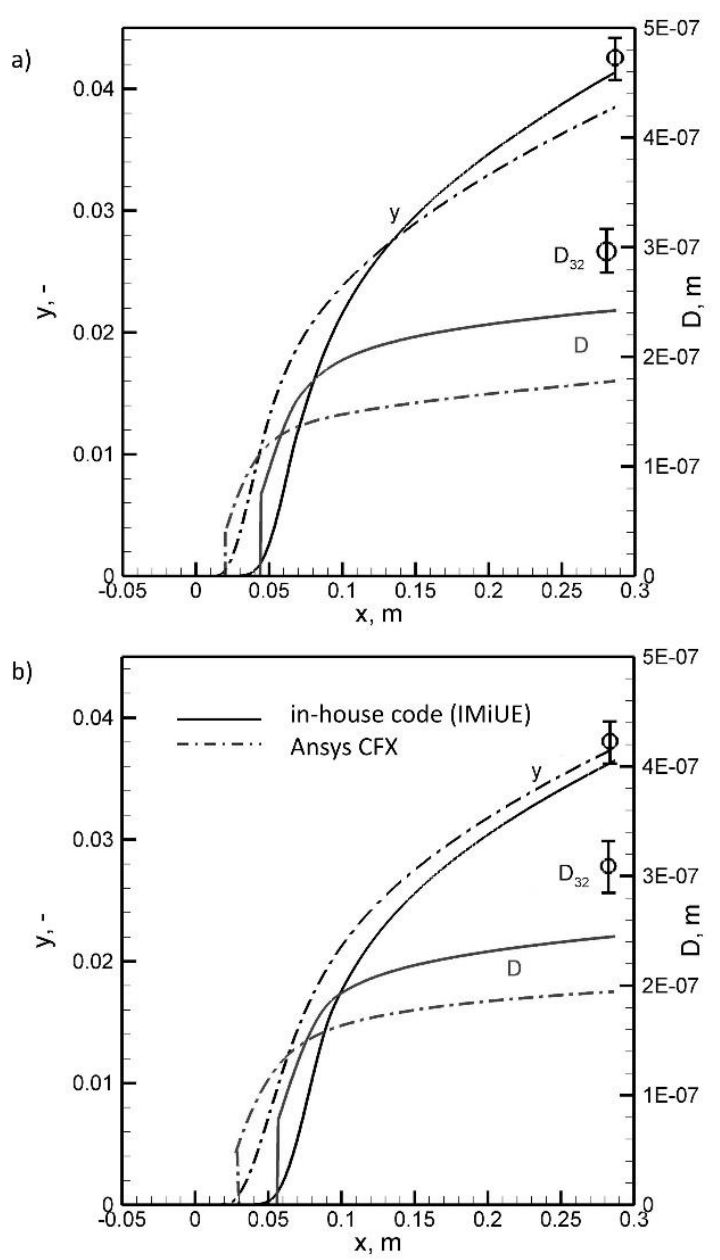

Fig. 10. Liquid phase parameter distribution along the nozzle for (a) $\mathrm{p}_{0}=124 \mathrm{kPa}, \mathrm{t}_{0}=118^{\circ} \mathrm{C}$ and (b) $\mathrm{p}_{0}=128 \mathrm{kPa}, \mathrm{t}_{0}=$ $121^{\circ} \mathrm{C}$ with Sauter mean diameter $D_{32}$ and $y$

Two measurement series were selected for analysis from many sessions of conducted tests. They differ in the total temperature at the inlet of the nozzle, at almost the same total pressure. In the first case, the values at the nozzle 
inlet are $p_{0}=124 \mathrm{kPa}$ and $\mathrm{t}_{0}=118^{\circ} \mathrm{C}$, in the second case $\mathrm{p}_{0}=128 \mathrm{kPa}$ and $\mathrm{t}_{0}=121^{\circ} \mathrm{C}$. For the first case (Fig. $10(a))$, comparing the wetness mass fraction and the average droplet size from measurements, in relation to both numerical codes, it can be observed that both determined values are higher than the values determined by Ansys CFX program and calculated by own numerical code developed at IMiUE. Similar relationships can be seen for the second selected case. Although for the degree of humidity the results of numerical tests and the experiment are quite similar, in the case of the Sauter diameter, the results of the experiment are about $50 \%$ higher, compared to the results of numerical calculations. However, even the results of two numerical analyzes alone in an interesting area show significant differences in the values of the obtained quantities. Although the tests performed in real flow conditions did not give significant results of quantitative tests, they showed additional technical and measuring imperfections of the constructed probe. Other obstacles having a significant impact on the quality of the results obtained were, for example, problems with determining the $I_{o}$ value during long-term tests and changing optical conditions during the flow of wet steam (dirt in the optics in the field of the light beam) or narrow water jets that pass through the optics, resulting from surface imperfections and the probe structure itself. This effect was also intensified by the pressure measuring impulse tube cleaning system, which allowed a temporary flow of atmospheric air into the tunnel.

\section{Conclusions}

The aim of the work was to develop a proprietary measurement procedure for a comprehensive method for determining the parameters of two-phase flow occurring in power machines mainly at the flow of wet steam in steam turbines.

The basic value, determined experimentally in the final phase, was the content of the liquid phase, defined as the wetness mass fraction of the medium. The design and testing procedure was described, as well as the tests in real conditions of the own probe $X$. For the probe, both static and dynamic tests were carried out at specially designed test stands. A comparative analysis of the correctness of the calculation method was carried out. Test results show that this method has good repeatability and reliability of obtained results under static conditions. Errors in determining the diameter size $D_{32}$ are in the range of $5.75 \%$ and the number of particles $12.1 \%$, which was considered good values for this type of measuring instruments, comparable to the error size of similar systems developed in other research centres. A calibration and functional check of the other temperature and pressure sensors included in the probe head were also carried out. In this case, it was also found that the flow velocity and angles were determined correctly, as well as the temperature values. During tests in real flow, the experiments showed the possibility of using the constructed probe for wet steam flow tests, the need to refine the mechanical design of the probe was noticed, and a slight discrepancy was obtained in the values obtained from the numerical analysis. However, it should be stated that both the results of real and static tests show that the developed measuring system is capable of measuring online the moisture content of steam in the IM iUE laboratory steam tunnel as well as the low-pressure steam turbine.

The use of determining $y$ using the light extinction technique with additional determined full thermodynamic parameters and flow visualization, e.g. with the schlieren technique, will allow the creation of a comprehensive measuring device enabling quick and simultaneous determination of the necessary amounts of wet steam flow.

In addition, to be able to carry out a more accurate anal ysis of losses in condensation steam flows, in further test tunnels it is desirable to further miniaturize the design of the probe $X$. U sing probe $X$ at the outlet of the blade palisade will allow more accurate determination of losses in the experiment and will also be very helpful in further work on the numerical model developed at IM iUE, including its validation.

The presented results were performed within the project TANGO 3 08/050/TAN19/0200 of The National Centre for Research and Development and Statutory Research Funds of the Silesian University of Technology.

\section{References}

1. Andreussi P., Di Donfrancesco A, and Messia M.(1988) An impedance method for the measurement of liquid hold-up in two-phase flow, Int. 1. Multiphase Flow, Vol. 14. p 777-785

2. Bachalo, W.D. and Houser, M.J. (1984) Phase Doppler spray analyzer for simultaneous measurements of drop size and velocity distributions, Optical Engineering, p.583-90

3. Blaszczuk A (2013) Experimental investigation of natural convection inside a upper part of vertical converging air channel using the Schlieren technique. Exp Thermal Fluid Sci, vol.50, p.178186.

4. Cai X. S., Wang N. N., Wei J. M, Zheng G.(1992), Experimental Investigation of the Light Extinction Method For Measuring Aerosol Size Distributions, J. Aerosol Sci., Vol.23, No. 7 p. 749-757

5. Cai X., Wanc L., Ouyang X., Pan Y. (1992), A Novel Integrated Probe System for Measuring the Two Phase Wet Steam Flow in Steam Turbine, Journal of Engineering Thermophisics, 22 (6), p. 52-57.

6. Dobbins, R.A. and Jizmagia, G.S. (1966): Particle size measurements based on use of mean scattering cross sections, Journal of the Optical Society of America

7. Doerffer P., Dykas S. (2005), Numerical analysis of shock induced separation delay by air humidity, Journal of Thermal Science, 14 (2), p. 120-125.

8. Dykas S. (2001), Numerical Calculation of the Steam Condensing Flow, TASK QUARTERLY 5, no. 4, p. 519-535. 
9. Dykas S., Majkut M., Smołka K., Strozik M. (2013), Research on Steam Condensing Flows in Nozzles with Shock Wave, Journal of Power Technologies, vol. 93, issue 5, p. 288-294.

10. Dykas S., Majkut M., Smołka K., Strozik M. (2015), Experimental Research on Wet Steam Flow With Shock Wave, Experimental Heat Transfer, vol. 28, issue 5, p. 417-429.

11. Dykas S., Majkut M., Smołka K., Strozik M. (2018), Analysis of the steam condensing flow in a linear blade cascade, Proc. Inst. Mech. Eng., A J. Power Energy, vol. 232 iss. 5, p. 501-514.

12. Dykas S., Majkut M., Strozik M., Smołka K. (2015), Experimental Study of Condensing Steam Flow in Nozzles and Linear Blade Cascade, International Journal of Heat and Mass Transfer, vol. 80, p. 5057.

13. Dykas S., Majkut M., Strozik M., Smołka K. (2015), Non-Equilibrium Spontaneous Condensation in Transonic Steam Flow Through Linear Cascade, Proceedings of the 11th European Conference on Turbomachinery.

14. Dykas S., Majkut M., Strozik M., Smołka K. (2018), An attempt to make a reliable assessment of the wet steam flow field in the de Laval nozzle., Heat Mass Transfer, p. 1-7

15. Dykas S., Majkut M., Strozik M., Smołka K. (2018), Comprehensive investigations into thermal and flow phenomena occurring in the atmospheric air twophase flow through nozzles, Int. J. Heat Mass Transf. 2017 vol. 114, p. 1072-1085

16. Dykas S., Smołka K., Majkut M., Strozik M. (2018), Identyfikacja pola przepływu pary mokrej w dyszach i płaskich palisadach łopatkowych, Wydawnictwo Politechniki Ślaskiej, Gliwice.

17. Eberle T., Schatz M. (2011), Experimental Study of Droplet Size and Wetness in a Model Steam Turbine Using Light Extinction Measurements, 25th Turbomachinery Workshop, Gdańsk/Krzeszna,

18. Horvath H. (2009), Gustav Mie and the scattering and absorption of light by particles: Historic developments and basics, Journal of Quantitative Spectroscopy \& Radiative Transfer, no. 110, p. 787799.

19. Kasprzyk, S. (1964): Ein Selbstansaugendes Kalorimeter zur Bestimmung der Dampffeucht, Brennstuff-Werme-Kraftwerk BWK, Vol. 16, p. 350355.

20. Kolovratník M., Bartoš O. (2015):CTU Optical probes for liquid phase detection in the $1000 \mathrm{MW}$ steam turbine, EPJ Web of Conferences 92

21. Ma, L., Sanders, S.T., Jeffries, J.B., Hanson, R.K. (2003): Monitoring and control of a pulse detonation engine using a diode-laser fuel concentration and temperature sensor, Proceedings of the Combustion Institute, vol. 29, p.161-166

22. Puzyrewski R. (1969), Theoretical and experimental studies on formation and growth of water drops in LP steam turbines, Transactions IFFM, 42-44, p. 289-303.

23. Puzyrewski R., Gardzilewicz A., Baginska M. (1973), Shock Waves in Condensing Steam Flowing
Through a Laval Nozzle, Archives of Mechanics, 25, no. 3, p. 393-409

24. Schatz M., Eberle T. (2014),Experimental study of steam wetness in a model steam turbine rig: presentation of results and comparison with computational fluid dynamics data, Proc IMechE Part A: J Power and Energy, Vol. 228(2) p. 129142

25. Su M.,, Xu F., Cai X., Ren K., Shen J.,(2007): Optimization of regularization parameter of inversion in particle sizing using light extinction method, China Particuology, Vol. 5, Iss. 4, p. 295299

26. Swithenbank, J., Beer, J.M., Taylor, D.S., Abbot, D., McCreath, G.C. (1977), A laser diagnostic technique for the measurement of droplet and particle size distribution, Progress in Aeronautics and Astronautics, vol. 53, p.421-447

27. Thurber, M.C. and Hanson, R.K. (2001), Simultaneous imaging of temperature and mole fraction using acetone planar laser-induced fluorescence, Experiments in Fluids, vol. 30, p. 93101

28. Twomney S. (1963): On the Numerical Solution of Fredholm Integral Equations of the First Kind by the Inversion of the Linear System Produced by Quadrature, Journal of the ACM (JACM), Vol. 10, Iss. 1, p. 97-101

29. White A.J. (1992), Condensation in steam turbine cascades, Department of Engineering, University of Cambridge. Praca doktorska.

30. Wróblewski W, Dykas S, Gepert A (2009): Steam Condensing Flow in Turbine Channels. Int $J$ Multiphase Flow vol. 35, Iss.6

31. Zhao, H. and Ladommatos, N.A. (1998): Optical diagnostics for in-cylinder mixture formation measurements in IC engines, Progress in Energy and Combustion Science, vol. 24, p. 297-336

32. Majkut, M. (2019): Zaawansowane techniki badań eksperymentalnych i wizualizacji przepływu gazów ściśliwych w maszynach energetycznych, Wydawnictwo Politechniki Ślaskiej, Gliwice 2019, ISBN 978-83-7880-623-3 